\title{
Phosphorylation et synthèse protéique au cours de la méiose de l'ovocyte
}

La protéine kinase $\mathrm{p} 34^{\text {cdc2 }}$ est une sous-unité du facteur MPF (M-phase promoting factor) qui contrôle la transition G2/M du cycle cellulaire. L'étude des divisions méiotiques de l'ovocyte de xénope a permis de caractériser deux substrats de la kinase $\mathrm{p} 34^{\text {cdc2 }}$ : une protéine de $47 \mathrm{kDa}$ (p47) identifiée comme la sous-unité $\gamma$ du facteur d'élongation de la synthèse protéique (EF1) et la sous-unité régulatrice de la caséine kinase II (CKII). La première protéine est phosphorylée par la kinase $\mathrm{p} 34^{\text {cdc2 }}$ pendant les divisions méiotiques des ovocytes, ce qui crée un lien direct entre l'activation de la kinase du MPF et la régulation de la synthèse protéique qui intervient lors de la méiose. La phosphorylation du second substrat indique que $\mathrm{p} 34^{\text {cdc2 }}$ peut être à l'origine d'une cascade de phosphorylations permettant d'expliquer les effets pléiotropiques du MPF. Ces résultats semblent pouvoir s'appliquer à d'autres modèles que les œufs d'amphibiens, notamment aux cellules de mammifères.

\section{Patrick Cormier \\ Odile Mulner-Lorillon Julia Morales \\ Robert Poulhe Robert Bellé}

\footnotetext{
ADRESSE

P. Cormier : Attaché temporaire d'Enseignement et de Recherche. O. Mulner-Lorillon : Directeur de Recherches, Cnrs. J. Morales : Boursière $M R T$, Université Paris 7. R. Poulhe : Assistant Ingénieur, Cnrs. R. Belle : professeur. Université Paris 6 . Physiologie de la reproduction, INRA, UA Cnrs 1449, université Pierre-et-Marie Curie, 4 place Jussieu, 75256 Paris Cedex 05, France.

'ovocyte de xénope est naturellement bloqué au stade prophase de la première division méiotique. La reprise des divisions est contrôlée par une hormone stéroïde, la progestérone (figure 1, p. 674). L'hormone induit le passage du stade prophase de la première division méiotique (prophase I) au stade métaphase de la deuxième division méiotique (métaphase II). A ce stade, la méiose est à nouveau bloquée jusqu'à la fécondation. L'ensemble de ces événements est appelé maturation méiotique.

$\mathrm{Au}$ cours de la transition prophase/métaphase apparaît le MPF, qui est le facteur ubiquitaire de con- trôle des divisions méiotiques et mitotiques ( $M$-phase promoting factor). Le $\mathrm{MPF}$ est un hétérodimère dont la sous-unité catalytique est une protéine kinase de $34 \mathrm{kDa}$ homologue au produit du gène $c d c 2$ de la levure $S$. pombe (cdc pour cell division control). Cette protéine kinase (p34 cdc2) possède in vitro une activité histone $\mathrm{H} 1$ kinase. La seconde sous-unité est une molécule de la famille des cyclines, protéines dont la concentration oscille en phase avec l'activité mitotique $[1,2]$.

En l'absence de stimulation hormonale, l'ovocyte de xénope contient le complexe kinase p34cdc2/cycline (MPF) sous une forme inactive appelée préMPF. Il est maintenu sous 


\section{RÉFÉRENCES}

1. Nurse P. Universal control mechanism regulating onset of a M-phase. Nature 1990 ; 344: 503-8.

2. Le Peuch C. La régulation de la division cellulairc. médecine/sciences $1990 ; 6$ : 10-7.

3. Gautier J, Solomon MJ, Booher RN, Bazan JF, Kirschner MW. cde25 is a spccific tyrosine phosphatase that directly activates p34cde2. Cell 1991; 67 : 197-211.

4. Srausfeld U, Labbé JC, Fesquet D, et al. Dephosphorylation and activation of a p34 cdc $2 /$ cyclin B complex in vitro by human CDC25 protein. Nature 1991 ; 365 : 242-5.

5. Ozon R, Mulner O, Boyer J, Bellé R Role on protein phosphorylation in xenopus oocyte meiotic maturation. In : Schlegel RA, Halleck MS, Rao PN, cds. Molecular Regulation of Nuclear Events in Mitosis and Meiosis. New York : Academic press, 1987 111-30.

6. Wasserman WJ, Richter JD, Smith I, I) Protcin synthesis during maturation promoting factor- and progesterone-induced maturation in xenopus oocytes. Dev Biol 1982 ; 89 : $152-8$

7. Mulner-Lorillon $\mathrm{O}$, Cormier P, Labbé $\mathrm{JC}$, et al. M-phase-specific cdc2 protein kinase phosphorylates the $\beta$ subunit of casein kinase II and increases casein kinase II activity. Eur J Biochem 1990 ; 193 : 529-34

8. Litchfield DW, Lozeman FJ, Cicirelli $\mathrm{MF}$, et al. Phosphorylation of the $\beta$ subunit of cascin kinasc II in human A431 cells. Identification of the autophosphorylation site and a site phosphorylated by p34 cde2. J Biol Chem 1991; 266 : 20380-9.

9. Cavadorc JC, Le Bouffant F, Labbé JC Les substrats de p34cde2, la kinase spécilique de la phase $\mathrm{M}$ du cycle cellulaire. médecine/sciences $1990 ; 6: 895-900$.

10. Gerhart J, Wu M, Kirschner M. Cell cycle dynamics of an M-phase-specific cytoplasmic factor in Xenopus lavis oocytes and cggs. J Cell Biol 1984; 98 : 1247-55.

11. Charbonncau M, Bonnec G, Boujard 1). Patterns of protein synthesis during xenopus oocyte maturation differ according to the type of stimulation. Cell Diff Dev $1990 ; 31$ : 197-206.

12. Swenson KI, Farrell KM, Ruderman JV. The clam embryo protein cyclin A induces entry into $\mathrm{M}$ phase and the resumption of meiosis in xenopus oocytes. Cell cettc forme par phosphorylation dc sites présents sur la sous-unité catalytique. L'activation du MPF est corréléc à la déphosphorylation de ces sites $[3,4]$

Lc MPF provoque les événements cytomorphologiques de la méiose : rupture de l'enveloppe nucléairc, condensation des chromosomes et misc cn place du fuscau métaphasique. Son apparition est associćc à dcux événements biochimiques majeurs : une augmentation du taux de phosphorylation des protéincs [5] et une augmentation de la synthèse des protéines [6].

L'identification des substrats de la kinase du MPF est indispensable pour comprendre comment ce factcur contrôlc ces ćvénements. Nous avons montré que la casćine kinase II, extraite d'ovaire de xénope, est phosphoryléc sur sa sous-unité régulatrice par la kinase du MPF et que cette phosphorylation provoque, in vitro, unc stimulation de l'activité casćine kinase [7]. Cette phosphorylation a récemment ćté retrouvéc sur l'enzyme purifićc à partir de ccllules humaines [8]. L'augmentation des phosphorylations, au moment de l'activation du MPF, résulte donc à la fois de la phosphorylation directe de différents substrats par la kinase du MPF [9] et ćgalement de l'activation en cascade de protéine kinases conduisant à la phosphorylation d'autres substrats. Ce processus permet d'amplifier et de moduler l'effet du MPF.
La voie conduisant à l'augmentation de la synthèse protéique après l'activation du MPF reste encore à ćlucider. Néanmoins, nous avons récemment identifić un factcur de la traduction comme ćtant un substrat physiologique de la kinasc du MPF, cc qui permet pour la première fois de proposer un modèle de régulation de la synthèse protéique au cours de la maturation.

\section{Synthèse protéique et divisions méiotiques}

La synthèse protćique est nćcessaire à la reprise des divisions méiotiques induite par la progestéronc. Ellc est indispensable à la première activation du MPF et est ćgalcment nécessaire à la réapparition du MPF lors de la dcuxième division méiotique [10].

L'analyse de la synthèse protéique au cours des divisions méiotiques de l'ovocyte (figure 2) montre que lc taux de synthèse globalc des protéines n'cst pas modifić entre la stimulation hormonale et l'activation du MPF. Il augmente ensuitc, pour attcindre en métaphase un niveau double de celui des ovocytes de prophase [6]. Ainsi l'augmentation de la synthèse protéique qui accompagne les divisions méiotiques intervient à un stade où ellc n'est plus nćcessaire à l'activation du MPF. Elle est cependant nćcessaire au bon déroulement des événements cytomorphologiques de la première division de la méiosc ainsi qu'à

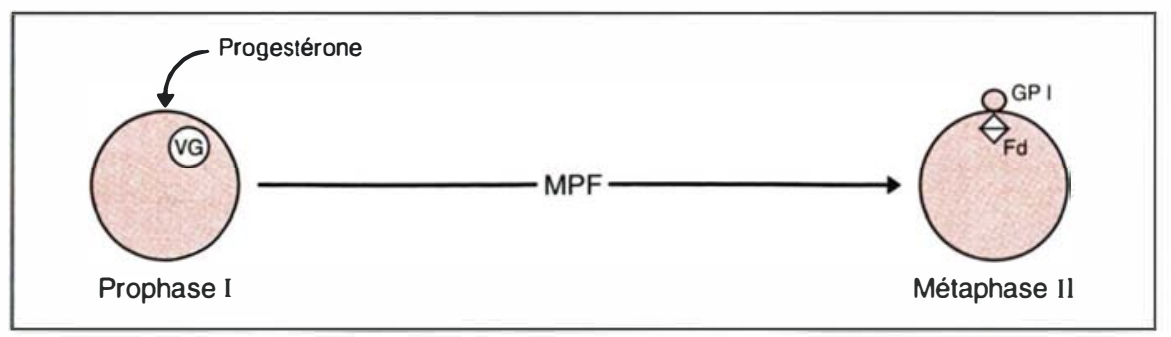

Figure 1. La maturation méiotique de l'ovocyte de xénope. La progestérone provoque la maturation méiotique de l'ovocyte. La maturation méiotique correspond au déroulement des événements de la méiose de la prophase I à la métaphase II. Au cours de la transition prophase/métaphase apparaît le facteur ubiquitaire de contrôle des divisions méiotiques et mitotiques: le MPF. Ayant achevé sa croissance, l'ovocyte se divise sans avoir à doubler de masse comme dans une mitose somatique. Le taux de synthèse protéique, qui est particulièrement bas dans l'ovocyte, peut donc être restreint aux protéines indispensables à la régulation des divisions cellulaires. VG : vésicule germinative; GP I: premier globule polaire; Fd : fuseau de division ; MPF : M-phase promoting factor ou maturation-promoting factor. 


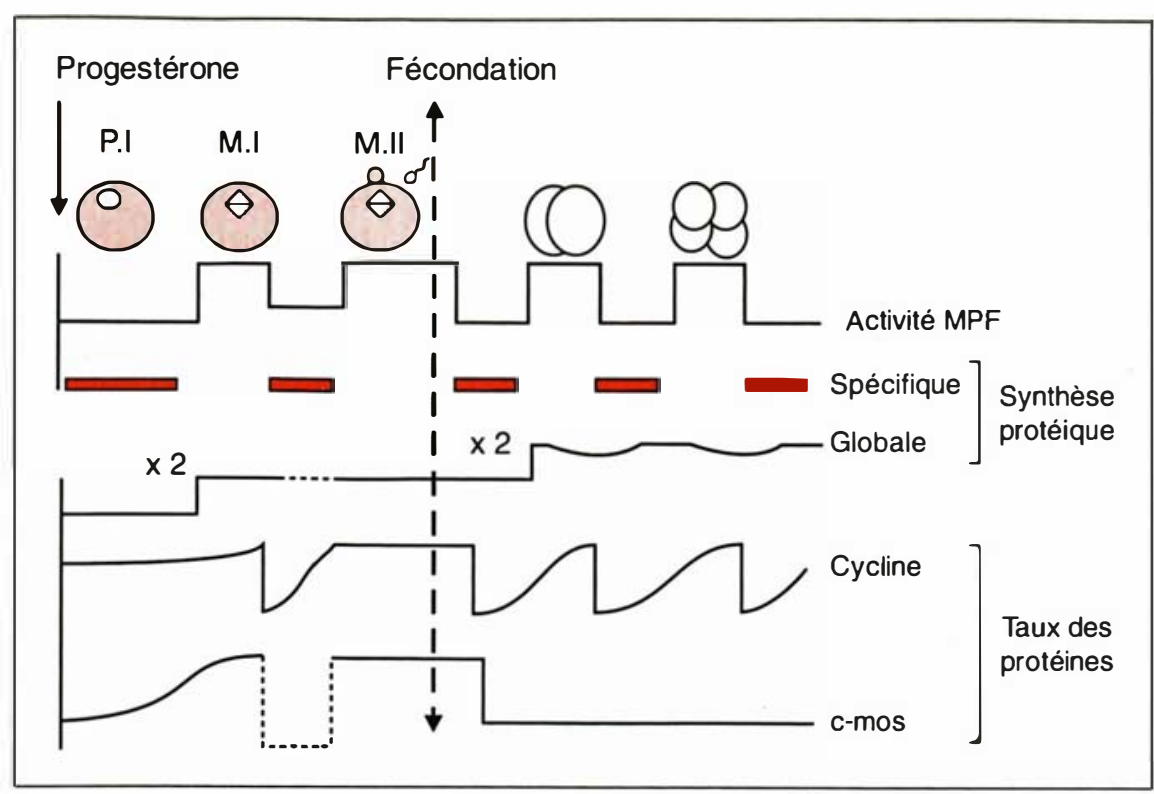

Figure 2. Activité MPF et synthèse protéique durant les divisions méiotiques et les deux premières divisions mitotiques. Le MPF actif possède in vitro une activité histone $\mathrm{H} 1$ kinase. Cette activité, indétectable dans les ovocytes en prophase, est maximale en métaphase. La synthèse protéique a été analysée durant les divisions méiotiques de l'ovocyte. Le taux de synthèse globale des protéines n'est pas modifié entre la stimulation hormonale et l'activation de MPF, puis il augmente d'un facteur deux en métaphase. Après fécondation, le taux de synthèse globale des protéines double à nouveau puis baisse légèrement à chaque mitose. La progestérone provoque une traduction spécifique d'un nombre limité de protéines telles les cyclines (dont la concentration oscille en phase avec l'activité mitotique) et le produit du proto-oncogène C-mos (voir texte). P.I : prophase I; M.I : métaphase I; M.II : métaphase II.

l'activation du MPF lors de la deuxième division.

L'analyse qualitative des protéines montre que l'augmentation résulte d'un accroissement du taux de synthèse globale et de l'apparition de nouvelles protéines. Au cours de la phase dépendante de la synthèse protéique, les modifications qualitatives sont faibles [11] et n'ont pu être identifiées à partir d'expériences classiques de marquage radioactif.

\section{Synthèses protéiques nécessaires à l'activation du MPF}

La caractérisation de protéines dont la synthèse est nécessaire pour l'activation du MPF après la stimulation hormonale n'a été possible qu'en étudiant l'effet de l'augmentation de la concentration dans l'ovocyte de diverses protéines susceptibles d'intervenir, en injectant soit l'ARN messager $m / s n^{\circ} 7$, vol. 8 , seplembre 92 tion spécifique de l'ARNm de p39 mos par la micro-in jection d'un oligonucléotide anti-sens inhibe l'activation du MPF par la progestéronc. L'hormone induit la synthèse de la protéine p39 mos dans l'ovocyte [17]. La micro-injection de l'ARNm codant pour p39mos, ou de la protéine ellc-même, induit l'activation du MPF en l'absence d'hormone [18-20].

Ces expériences montrent que l'introduction par micro-injection de protéines spécifiques (cyclines ou p39 ${ }^{\text {mos }}$ ) pcut induire la maturation et l'activation de MPF. Leur faible concentration intracellulaire (inférieurc à $1 / 1000$ des protéines cytosolubles) explique pourquoi elles n'ont pas été détectées par des expériences de marquage radioactif. La progestérone provoque donc la traduction d'un nombre limité de protéines, dont au moins une (p39 mos $)$ est indispensable à l'action hormonale.

Ainsi, le mécanisme d'action de la progestérone serait de provoquer la traduction d'ARNm spécifiques au cours de la reprise de la première division méiotique (figure $3, p .676$ ). La (ou les) protéine(s) synthétisée(s) entraîne(nt) alors l'activation du MPF. Lorsque celui-ci est activé, il provoque une augmentation de la synthèse protéique qui comprend aussi une traduction de nouveaux messagers, dont certains joueraient un rôle lors de la division suivantc. téine elle-même. Ainsi, la microinjection de l'ARNm codant pour une des cyclines mitotiques (cycline A ou cycline B) provoque l'activation du MPF [12, 13]. Le même effet est obtenu par la micro-injection de la protéine elle-même [14]. La progestérone provoque effectivement une augmentation de la synthèse des différentes cyclines [15]. Cependant, il n'est pas démontré que la synthèse des cyclines soit indispensable dans le mécanisme d'action hormonal. Les expériences de micro-injection de cyclines sont difficilement interprétables puisque l'ovocyte en prophase contient du pré-MPF sous forme d'un complexe kinase $\mathrm{p} 34^{\mathrm{cdc} 2} / \mathrm{cy}$ cline B inactif $[15,16]$.

Les mêmes techniques ont permis de montrer que le produit du protooncogène $c$-mos, la protéine p39 mos, est aussi impliqué dans les étapes précoces de la maturation. La destruc-

\section{Régulation de la synthèse protéique}

L'ovocyte en fin de croissance contient une réserve très importante de messagers maternels et de ribosomes dont une très faible proportion est engagée dans la synthèse protéique [21]. D'une part, les ARNm sont stockés sous forme de ribonucléoprotéines (mRNP) inaccessibles à la traduction et, d'autre part, la machinerie de la traduction est fortement inhibée [22].

Le mécanisme de l'augmentation de la synthèse protéique, au cours des divisions méiotiques induites par l'hormone, n'est pas connu. Deux médiateurs ont été proposés : les ions $\mathrm{H}^{+}$et les ions $\mathrm{Mg}^{2+}$. Ainsi, une augmentation du $\mathrm{pH}$ intracellulaire a lieu au moment de l'apparition du 


\section{RÉFÉRENCES}

13. Pines J, Hunt $T$. Molecular cloning and characterization of the mRNA for cyclin from sca urchin eggs. EMBO J $1987 ; 6$ : 2987-95.

14. Roy LM, Swenson KI, Walker DH, et al. Activation of $\mathrm{p} 34^{\mathrm{cdc} 2}$ kinase by cyclin A. J Cell Biol 1991; 113 : 507-14.

15. Kobayashi H, Minshull J, Ford C, Golsteyn R, Poon R, Hunt $T$. On the synthesis and destruction of A- and B-type cyclins during oogenesis and meiotic maturation in Xenopus lavis. J Cell Biol 1991; 114: 755-65.

16. Gautier J, Maller JL. Cyclin B in xenopus oocytes : implications for the mechanism of pre-MPF activation. EMBOJ 1991; 10 : 177-182.

17. Sagata $\mathbf{N}$, Oskarsson $M$, Copeland $T$, Brumbaugh J, Vande Woude GF. Function of $c$-mos proto-oncogene product in meiotic maturation in xenopus oocytes. Nature 1988 335 : 519-25.

18. Sagata N, Daar I, Oskarsson M, Showalter SD, Vande Woude GF. The product of the mos proto-oncogene as a candidate "initiator " for oocyte maturation. Science 1989 ; 245 : 643-6.

19. Frceman RS, Pickham KM, Kanki JP, Lee BA, Pena SV, Donoghuc DJ. Xenopus homolog of the mos protooncogene transforms mammalian fibroblasts and induces maturation of xenopus oocytes. Proc Natl Acad Sci USA 1989 ; 86 : 5805-9.

20. Yew N, Mellini ML, Vande Woude GF. Meiotic initiation by the mos protein in xenopus. Nature $1992 ; 355: 649-52$.

21. Woodland HR. Changes in the polysome content of developing Xenopus lavis embryos. Dev Biol 1974; 40 : 90-101.

22. Laskey RA, Mills AD, Gurdon JB, Partington GA. Protein synthesis in oocytes of Xenopus lavis is not regulated by the supply of messenger RNA. Cell 1977 ; 11 : 345-51.

23. Stith BJ, Maller JL. Increased intracellular $\mathrm{pH}$ is not necessary for ribosomal protein S6 phosphorylation, increased protein synthesis, or germinal vesicle breakdown in xenopus oocytes. Dev Biol 1985; 107 : 460-9.

24. Horowitz SB, Tluczek LJM. Gonadotropin stimulates oocyte translation by increasing magnesium activity through intracellular potassium-magnesium exchange. Proc

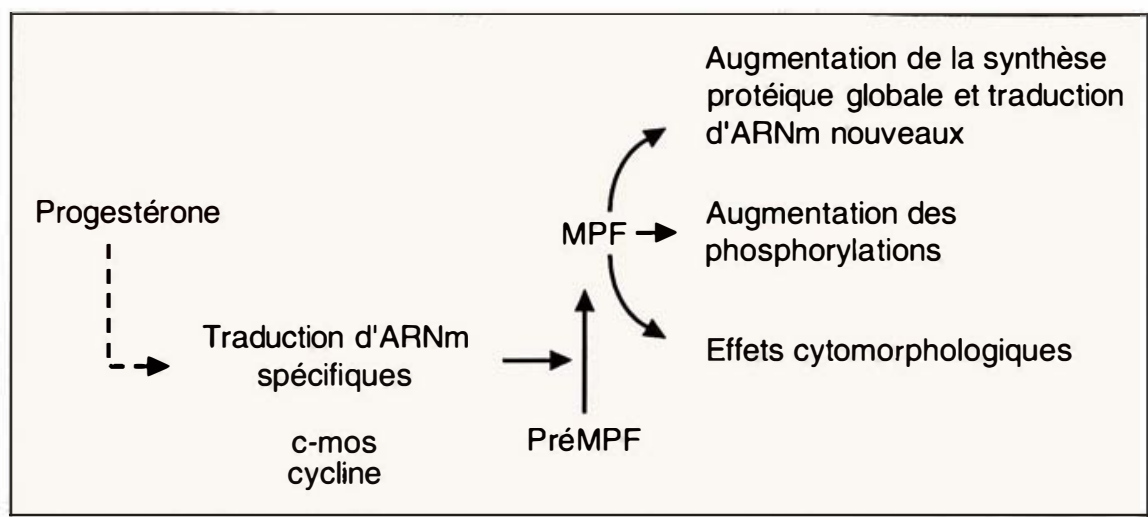

Figure 3. Schéma du mécanisme d'action de la progestérone. La progestérone provoque une lecture d'ARNm spécifiques au cours de la première division méiotique (voir texte). Lorsque le MPF est activé, il entraîne une augmentation de la synthèse protéique qui comprend aussi une traduction de nouveaux messagers, dont certains joueraient un rôle lors de la division suivante.
MPF et cette augmentation est capable de doubler le taux de synthèse protéique. Cette hypothèse doit toutefois être écartée puisque l'augmentation de $\mathrm{pH}$ n'est pas nécessaire à l'augmentation de la synthèse protéique [23]. Par ailleurs, le taux de synthèse protéique dépend de la concentration intracellulaire en $\mathrm{Mg}^{2+}$. Une augmentation de la concentration en ions $\mathrm{Mg}^{2+}$ pourrait être pro- voquée par l'augmentation des ions $\mathrm{K}^{+}$[24]. Cependant, aucune augmentation de $\mathrm{Mg} 2+$ intracellulaire n'a été mise en évidence au cours des divisions méiotiques.

L'augmentation de la synthèse protéique au cours des divisions méiotiques est corrélée à une augmentation de trois fois de la proportion de ribosomes présents dans les polysomes [21]. Cela implique qu'il existe un recru-

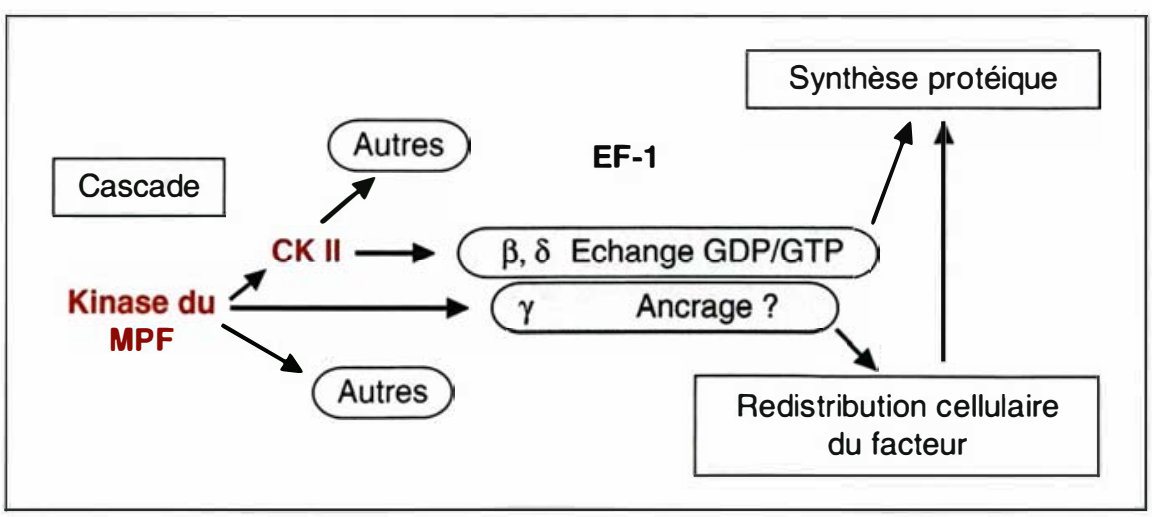

Figure 4. Hypothèse du contrôle de la synthèse protéique par la kinase du MPF. Nos résultats permettent de proposer un schéma du contrôle de la synthèse protéique par la phosphorylation des protéines au cours des divisions méiotiques. Le MPF peut agir sur le mécanisme de la phase d'élongation de la synthèse protéique en phosphorylant directement la sous-unité $\gamma$ du facteur d'élongation EF1, mais aussi les sous-unités échangeuses de GDP/GTP, par l'intermédiaire de son action sur la caséine kinase II (CKII). La sous-unité y pourrait jouer un rôle d'ancrage du complexe EF1 et la phosphorylation de cette protéine d'ancrage serait le signal de la redistribution intracellulaire du facteur d'élongation de la synthèse protéique EF1. 
tement d'ARNm pendant la maturation, soit par démasquage [25], soit par changement de localisation. En effet, les ARNm ne sont pas tous distribués de façon uniforme dans l'ovocyte. Certains sont localisés, tel $\mathrm{Vg} 1$, au niveau de l'hémisphère végétatif [26] et, au cours des divisions méiotiques, il existe une redistribution d'ARNm [27]. Cependant, le recrutement d'ARNm n'est pas suffisant pour expliquer l'augmentation de la synthèse protéique puisque la traduction d'ARNm exogènes dans des ovocytes en prophase se fait au détriment de celle des ARNm endogènes, cela résultant de l'inhibition de la machinerie de la traduction (voir cidessus). Puisque l'activité spécifique des facteurs d'initiation et d'élongation de la synthèse protéique n'est pas modifiée au cours des divisions méiotiques [28], un des facteurs régulateurs de la synthèse protéique serait la disponibilité des éléments de la machinerie de la traduction. Cette hypothèse est plausible puisque certains facteurs nécessaires à la synthèse protéique, comme les facteurs d'élongation, sont distribués dans différents compartiments de la cellule $[29,30]$.

\section{MPF, phosphorylations et synthèse protéique}

Les signaux responsables des changements de la machinerie de traduction sont induits par le MPF dont la sousunité catalytique est une protéine kinase. Plusieurs protéines intervenant dans le mécanisme de traduction peuvent être contrôlées par phosphorylation/déphosphorylation. Ce sont des protéines régulatrices de l'initiation et de l'élongation, des protéines ribosomiques ainsi que des enzymes nécessaires à l'élongation telles que les aminoacyl-ARNt synthétases [31]. Parmi les protéines phosphorylables impliquées dans le contrôle de la synthèse protéique, nous avons montré que la sous-unité $\gamma$ du facteur d'élongation EF1 (elongation factor 1) est phosphorylée in vivo par la kinase du MPF au cours de la maturation [32, 33]. Deux sites potentiels de phosphorylation (KTPE et TPKK) sont présents, respectivement en positions 46 et 230 , dans la séquence protéique déduite de celle de l'ADNc.

$\mathrm{m} / \mathrm{s} n^{\circ} 7$, vol. 8 , septembre 92
De plus, deux des sous-unités du facteur d'élongation EF1, les sous-unités $\beta$ et $\delta$ qui possèdent une activité d'échange GDP/GTP nécessaire à la phase d'élongation, sont des substrats de la caséine kinase II [29]. Cette kinase est phosphorylée et activée par la kinase du MPF. Celle-ci pourrait donc contrôler, par l'intermédiaire de la caséine kinase II, les sous-unités échangeuses de GDP/GTP de la phase de l'élongation de la synthèse protéique et ainsi agir sur le mécanisme de la traduction.

Au cours des divisions méiotiques de l'ovocyte de xénope, parmi les protéines dont le taux de phosphorylation augmente, deux, au moins, sont impliquées dans la synthèse protéique : la protéine ribosomique $\mathrm{S} 6$ et le facteur d'élongation EF1. La phosphorylation de la protéine ribosomique S6 permettrait le recrutement des ribosomes dans les polysomes [35]. Cependant, dans l'ovocyte, l'augmentation de la phosphorylation de S6 peut avoir lieu sans augmentation de la synthèse protéique et, inversement, l'augmentation de la synthèse protéique peut intervenir sans augmentation de la phosphorylation de S6 [23]. Aussi le rôle de la phosphorylation de S6 au cours de la maturation reste encore à élucider.

La mise en évidence de la sous-unité $\gamma$ du facteur d'élongation EF1 comme cible physiologique du MPF constitue un lien direct entre l'activité MPF et la machinerie de la traduction. La phosphorylation de cette protéine pourrait être un signal de changement d'activité d'un des éléments du complexe EF1 et aboutir non seulement à une augmentation du niveau global de la synthèse protéique, mais peut-être aussi à une lecture différentielle des ARNm [36, 37].

La phosphorylation de la sous-unité $\gamma$ de EF1 par le MPF est en effet corrélée à un changement de localisation de ce complexe au cours de la maturation [29]. Cela suggère que cette sous-unité pourrait jouer un rôle de protéine d'ancrage du complexe et que sa phosphorylation pourrait être un signal de redistribution intracellulaire du facteur d'élongation, contrôlant ainsi localement la synthèse protéique. Tous ces mécanismes ne sont d'ailleurs probablement pas limités aux œufs d'amphibiens ; nous avons, en effet, récemment montré que la sous-unité $\gamma$ du complexe EF1 isolé de foie de lapin est, elle aussi, un substrat de la kinase du MPF (résultats personnels non publiés), ce qui suggère que la phosphorylation de EF1 intervient également au cours des divisions mitotiques de cellules somatiques de vertébrés supérieurs. A partir de ces données, on peut proposer un modèle du contrôle de la synthèse protéique par le MPF, relayé notamment par EF1 et la caséine kinase II (figure 4). Des études ultérieures permettront de tester ce modèle, probablement trop simple dans l'état actuel de nos connaissances, et de déterminer son application à différentes espèces et à différents types de cellules

\section{Summary}

Phosphorylation and protein synthesis during oocyte'meiosis

During the past few years, both genetic studies in yeast and biochemical analysis in oocytes, have lead to the characterization of a universal protein kinase involved in the regulation of cell division control. This protein kinase is called $\mathrm{p} 34^{\mathrm{cdc} 2}$, it phosphorylates preferentially histone $\mathrm{H} 1$ in vitro, and is part of a heterodimer which constitutes in association with a protein of the cyclin family, the so-called MPF or Mphase promoting factor. Using meiotic maturation of xenopus oocytes as a model, we have characterized two substrates of p34 ${ }^{\text {cdc2 }}$ kinase activity. One has been identified to the $\gamma$ subunit of a macromolecular complex known to be implicated in peptide chain elongation, called elongation factor 1 (EF1). This $\mathrm{p} 47$ protein $(\mathrm{EF} 1 \gamma)$ is phosphorylated by $\mathrm{p} 34^{\mathrm{cdc} 2}$ during the course of meiotic cell division and constitutes for the first time a direct

$\rightarrow$ (Suite p. 678) 


\section{RÉFÉRENCES}

25. Sommerville J. RNA-binding phosphoproteins and the regulation of maternal mRNA in xenopus. J Reprod Fert $1990 ; 42$ (suppl) : 225-33.

26. Melton DA. Translocation of a localized maternal mRNA to the vegetal pole of xenopus oocytes. Nature $1987 ; 328$ : 80-2.

27. Capco D, Jeffery WR. Transient localizations of messenger RNA in Xenopus lavis oocytes. Dev Biol $1982 ; 89: 1-12$.

28. Richter JD, Wasserman WJ, Smith LD. The mechanism for increased protein synthesis during xenopus oocyte maturation. Dev Biol 1982 ; 89 : 159-67.

29. Bellé R, Cormicr P, Poulhe R, Morales J, Huchon D, Mulncr-Lorillon O. Protein phosphorylation during meiotic maturation of xenopus oocytes : cdc2 protcin kinase targets. Int J Dev Biol $1990 ; 34$ : 111-5.

30. Riis B, Rattan SIS, Clark BFC, Merrick WC. Eukaryotic protein elongation factors. Trends Biol Sci $1990 ; 15$ : 420-4.

31. Hershcy JWB. Translational control in mammalian cells. Annu Rev Biochem 1991 ; $60: 717-55$

32. Mulner-Lorillon $\mathrm{O}$, Poulhe $\mathrm{R}$, Cormicr P, Labbé JC, Doréc M, Bcllé R. Purification of a 447 phosphoprotein from Xenopus lavis oocytes and identification as an in vivo and in vitro p34 cdc2 substratc. FEBS Lett $1989 ; 251: 219-24$

33. Bcllé R, Derancourt J, Poulhe R, Capony JP, Ozon R, Mulner-Lorillon O. A purified complex from xenopus oocytes contains a $\mathrm{p} 47$ protcin, an in vivo substrate of $\mathrm{MPF}$, and a p30 protein respectively homologous to clongation factors $\mathrm{EF}-1 \gamma$ and EF-13. FEBS Lett 1989 ; 255 : 101-4.

34. Cormier P, Osborne HB, Morales J, et al. Molccular cloning of xenopus clongation factor $1 \gamma$, major $M$-phase promoting factor substratc. Nucleic Acids Res 1991; 19 : 6644.

35. Thomas G, Perez JM, Sicgmann M, Otto AM. The effect of serum, EGF, PGF $2 \alpha$ and the initiation of protein and insulin on S6 phosphorylation and the initiation of protein and DNA synthesis. Cell $1982 ; 30: 235-42$

36. Ryazanov AG, Rudkin BB, Spirin AA. Regulation of protein synthesis at the clongation stage. New insights into the control of gene expression in cukaryotes. FEBS Lett $1991 ; 285: 170-5$

37. Gchrke L, Bast RE, Han J. An analysis of rates of polypeptide chain elongation in avian liver cxplants following in vivo estrogen treatment. J Biol Chem 1981; 256 : 2522-30.

\section{Summary (suite)}

link between $\mathrm{p} 34^{\mathrm{cdc} 2}$ activation and regulation of protein synthesis which occurs during meiosis. The second substrate of $\mathrm{p} 34^{\text {cdc2 }}$ is a biochemically well known protein kinase, casein kinase II, which happens to be phosphorylated on its regulatory subunit, and consequently activated. This result provides biochemical explanation to the burst in protein phosphorylation associated with p34 cde2 activation. Furthermore, its provides some clues to the analysis of the pleiotropic effects of $\mathrm{p} 34^{\mathrm{cdc} 2}$ protein kinase. We discuss in the present report our findings on $\mathrm{p} 34^{\text {cdc2 }}$ protein kinase substrates using xenopus oocytes, in the light of their relationship with the regulation of protein synthesis. We propose that our discoveries could be of much wider interest than in oocyte meiosis since recent data from ourselves and others indicate that p34 cdc2 kinase can phosphorylate elongation factor 1 and casein kinase II from other sources than xenopus oocytes, including homologous proteins from mammalian somatic cells.
TIRÉS A PART

P. Cormicr 